\title{
Repercussões psicossociais do câncer na infância e na adolescência
}

\author{
Lucas Rossato* \\ Ana María Ullán De La Fuente** \\ Fabio Scorsolini-Comin***
}

\begin{abstract}
Resumo
Trata-se de uma revisão narrativa com objetivo de evidenciar repercussões psicossociais do câncer na vida de crianças/ adolescentes adoecidos e familiares/cuidadores. Os dados encontrados na literatura científica demonstram que o câncer é um evento complexo e amplo, marcado por determinantes subjetivos e sociais, sendo os aspectos psicológicos, financeiros, disparidades no acesso ao tratamento em contexto global e a escolarização, socialização as principais dimensões que sofrem interferência do processo de adoecimento/tratamento. A compreensão das implicações psicossociais torna-se essencial para a prestação de um cuidado integral em saúde, uma vez que esse evento afeta o modo de vida da pessoa adoecida e a dinâmica familiar.
\end{abstract}

Palavras-chave: Infância; Adolescência; Câncer em Crianças; Psico-oncologia pediátrica; Psico-oncologia.

\section{Psychosocial repercussion of cancer in childhood and adolescence}

\begin{abstract}
It is a narrative review with the objective of showing psychosocial repercussions of cancer in the lives of sick children/adolescents and family members/caregivers. The data found in the scientific literature demonstrate that cancer is a complex and broad event, marked by subjective and social determinants, with psychological, financial aspects, disparities in access to treatment in a global context and schooling, socialization the main dimensions that suffer interference of the illness/treatment process. Understanding the psychosocial implications becomes essential for the provision of comprehensive health care, since this event affects the sick person's way of life and family dynamics. Keywords: Childhood; Adolescence; Cancer in children; Pediatric psycho-oncology; Psycho-oncology.
\end{abstract}

* ORCID iD http://orcid.org/0000-0003-3350-0688. Universidade de São Paulo. Escola de Enfermagem de Ribeirão Preto. Programa de Pós-Graduação em Enfermagem Psiquiátrica. Doutorando no Programa de Pós-Graduação em Enfermagem Psiquiátrica na Escola de Enfermagem de Ribeirão Preto (EERP) da Universidade de São Paulo - USP. Bolsista de Pós-Graduação da Coordenação de Aperfeiçoamento de Pessoal de Nível Superior - CAPES. Graduado em Psicologia pela Universidade Federal de Goiás - Regional Jataí (2013) e mestre em Psicologia pela Universidade Federal do Triângulo Mineiro (2017), com bolsa concedida pela Fundação de Amparo à Pesquisa do Estado de Minas Gerais (FAPEMIG). Integrante do ORÍ - Laboratório de Pesquisa em Psicologia, Saúde e Sociedade da EERP-USP e do Grupo de Pesquisa Teoria Histórico-Cultural e Processos Psicossociais da Universidade Federal de Catalão. E-mail: rossatousp@usp.br.

** Universidad de Salamanca. Departamento de Psicología Social y Antropología. Graduada e Doutora em Psicologia pela Universidade de Salamanca. Docente vinculada ao Departamento de Psicologia Socdial e Antropologia da Universidade de Salamanca e do Programa de Pós-Graduação em Psicologia. E-mail: ullan@usal.es .

*** ORCID iD http://orcid.org/0000-0001-6281-3371. Universidade de São Paulo. Escola de Enfermagem de Ribeirão Preto. Programa de Pós-Graduação em Enfermagem Psiquiátrica. Professor do Departamento de Enfermagem Psiquiátrica e Ciências Humanas da Escola de Enfermagem de Ribeirão Preto da Universidade de São Paulo (EERP-USP) e do Programa de Pós-Graduação em Enfermagem Psiquiátrica da EERP-USP. Bolsista de Produtividade em Pesquisa do CNPq. Psicólogo (Bacharelado, Licenciatura e Formação de Psicólogo) pela Faculdade de Filosofia, Ciências e Letras de Ribeirão Preto da Universidade de São Paulo (FFCLRP-USP). Mestre e Doutor em Psicologia pela Universidade de São Paulo (USP). Realizou dois Pós-Doutorados na Universidade de São Paulo (2013-2015 e 2016-2017), na área de Tratamento e Prevenção Psicológica (ambos com Bolsa PDJ-CNPq). Líder do ORÍ - Laboratório de Pesquisa em Psicologia, Saúde e Sociedade, ligado à EERP-USP. E-mail: fabio.scorsolini@usp.br . 


\section{Introdução}

O câncer infantojuvenil é um problema global de saúde pública que tem requerido atenção em níveis nacionais e internacionais (Siegel, Miller \& Jemal, 2018). Apesar dos avanços significativos nas formas de tratamento nos últimos anos, ainda assim é uma das principais causas de morte na faixa etária de 1 a 19 anos. Em muitas situações, o diagnóstico oportuno do câncer em crianças é dificultado devido à raridade da doença e à apresentação inespecífica dos sintomas (Dang-Tan \& Franco, 2007).

Os diferentes fatores envolvidos no adoecimento por câncer na infância e adolescência fazem com que essa doença se caracterize por ser um evento marcante no processo de desenvolvimento, interferindo nos aspectos físicos, cognitivos e emocionais da pessoa acometida pela doença e dos sujeitos que acompanham o processo de tratamento. Por ser uma doença rara antes dos 20 anos de idade, quando ocorre levanta uma série de preocupações médicas, psicológicas, éticas e sociais (Steliarova-Foucher, Colombet, Ries, Moreno, Dolya, Bray, Hesseling, Shin, Stiller \& Tretarre, 2017) que precisam ser problematizadas.

O adoecimento por câncer, em muitas situações, é visto como algo que subverte o que se espera em termos do desenvolvimento humano para a infância e adolescência, haja vista que doenças de caráter crônico geralmente são esperadas para pessoas adultas ou idosas. Devido às dimensões que assume, consiste em uma experiência que provoca impactos na vida para os pacientes, famílias e profissionais de saúde envolvidos no cuidado e tratamento (Galligan, 2017; Manoharan \& O’Brien, 2020).

O imaginário social construído ao longo dos anos em relação à doença contribui para que a mesma seja considerada uma grave forma de adoecimento envolta de sofrimento, limitações e procedimentos difíceis de serem enfrentados, assim como a iminência da morte. Assim, o impacto do câncer acaba gerando medo e ansiedade associados a outras limitações da patologia, principalmente quando os pacientes entram em contato com a realização de exames invasivos e dolorosos.

Os procedimentos a serem realizados na maioria das vezes são desconhecido pelos pacientes e familiares e, embora haja explicações por parte da equipe de saúde em relação ao que será realizado, como será realizado e as sensações que podem ocorrer, ainda assim podem ser propulsores para o surgimento de medos, fantasias e temores. Além disso, as repercussões de um diagnóstico de câncer e o tratamento subsequente podem ser ainda mais prejudiciais na infância e adolescência (Yi, Kim \& Sang, 2016).

Compreende-se que o adoecer por câncer na infância e adolescência coloca em suspensão todo o imaginário social relacionado ao que se espera da vida das pessoas nesta fase do desenvolvimento (Gomes, Lima, Rodrigues, Lima \& Collet, 2013). Além disso, uma série de mudanças passarão a incidir, promovendo rupturas no modo de vida e atividades cotidianas, bem como no desenvolvimento típico esperado para essa etapa.

As implicações psicossociais do adoecimento e enfrentamento do câncer infantojuvenil são diversas e importantes de serem consideradas pelos profissionais de saúde que atuam com essa população, a fim de compreender as vivências dos sujeitos, bem como planejar estratégias de intervenção que considerem esses aspectos. Nesse sentido, o objetivo desse trabalho é sumarizar os principais impactos psicossociais do câncer nas vivências de crianças e adolescentes adoecidas relatados na literatura científica.

\section{Método}

Trata-se de uma revisão narrativa da literatura científica. Os artigos de revisão narrativa são publicações amplas, que procuram descrever e discutir o desenvolvimento de um assunto, sob ponto de vista teórico e/ou contextual (Rother, 2007). A revisão narrativa é mais abrangente justamente por permitir a inclusão de referências com diferentes níveis de evidência e localizadas a partir de diferentes métodos de busca. O foco desse tipo de revisão está justamente em permitir a atualização em relação a determinado conhecimento sem que os mecanismos controlados de busca possam excluir registros importantes, mas que, porventura, não atendam aos critérios de elegibilidade que, muitas vezes, mostram-se inadequados em relação às investigações em determinados campos. Os aspectos psicossociais elencados como relevantes para a compreensão do cenário em tela foram: diagnóstico, tratamento, abandono do tratamento e implicações psicossociais. Esses vértices serão discutidos visando a compor um rol de conhecimentos acerca dos principais aspectos psicossociais associados ao câncer infanto-juvenil.

\section{Resultados e Discussão}

Diversas são as implicações psicossociais que o câncer infantojuvenil acarreta na vida das pessoas adoecidas 
e de seus familiares e cuidadores durante o diagnóstico, o tratamento e pós-tratamento. Observa-se que aspectos de ordem social, emocional/psicológicos, subjetivos, educacionais e econômicos podem perpassar as vivências dessa doença e são importantes de serem considerados.

\section{Repercussões psicológicas}

O impacto psicológico do câncer inicia-se geralmente no momento em que é comunicado o diagnóstico e devem ser considerados na atenção e cuidado ofertados pelos serviços de saúde, uma vez que interferirão no modo como as crianças, os adolescentes e seus familiares/cuidadores irão lidar com a doença. Na maioria das situações, os pacientes e seus familiares/cuidadores não estão preparados para receberem o diagnóstico da doença e quando a recebem, emerge todo o imaginário social construído historicamente. Embora os principais efeitos do adoecimento por câncer estejam relacionados ao impacto desta doença no organismo das pessoas, o imaginário social é carregado de significados que interferirão na saúde mental dos sujeitos adoecidos e das pessoas que o acompanham durante o adoecimento.

O processo de revelação do câncer infantojuvenil é demarcado como sendo um momento difícil de ser enfrentado pela criança e adolescente e pelos familiares/ cuidadores que se deparam com uma doença socialmente temida, incerta e que impactará nos contextos de vida de todos. Deste modo, o diagnóstico e o tratamento do câncer podem ser considerados uma forma de trauma e, como tal, impactam o desenvolvimento e o bem-estar psicológico das pessoas envolvidas com esta situação de adoecimento (Kosir, Wiedemann, Wild \& Bowes, 2019).

Ao receber o diagnóstico de câncer, as crianças e as famílias percebem que seu mundo como o conheciam mudará para sempre. Segundo Woodgate (2006), familiares relatam sentimentos de que algo muito ruim e assustador pode acontecer, que o mundo não será mais o mesmo a partir daquele momento e as perspectivas futuras ficam suspensas nas incertezas advindas da doença, elevando o diagnóstico de câncer como um momento decisivo em suas vidas.

As implicações psicológicas do tratamento surgem devido a todo contexto de situações difíceis de serem enfrentadas e que fazem parte do percurso do adoecer. Sabe-se que pacientes com câncer na infância podem sofrer estresse significativo como resultado do tratamento ou da própria doença (Kosir, Wiedemann, Wild \& Bowes,
2019). Além disso, ter que lidar com a possibilidade do fim da vida ou com a concretude da finalização desta, faz do câncer um evento temido por todos os envolvidos. No caso dos familiares e cuidadores, a literatura científica tem exposto que o momento do diagnóstico pode gerar sofrimento psíquico, sendo que o sofrimento parental pode afetar a criança e a dinâmica familiar (Rattan, 2020).

Como qualquer estressor grave, a maneira pela qual uma criança é afetada pelo câncer e responde psicologicamente varia com a idade (Kurtz \& Abrams, 2011), suporte que recebe dos familiares, capacidade de compreender a doença, tipo do câncer, impacto na imagem corporal, apoio social, ajustamento ao tratamento e a novas rotinas, etc. (Steinmetz \& Tarquinio, 2018). Aspectos contextuais, como acesso a serviços de saúde e tratamentos adequados, podem igualmente interferir nesse processo.

Entre os aspectos estressores relacionados câncer também devem ser destacados os efeitos adversos para as crianças/adolescentes, que incluem: dor, perda de cabelo, picadas de agulha, remédios desagradáveis, visitas ao hospital e/ou passar a noite no hospital e dificuldades para dormir (Carrion-Plaza, Jaen \& Montoya-Castilla, 2020; Hildenbrand, Clawson, Alderfer \& Marsac, 2011; Leandro, Silva, Lopes, Guedes, Nunes, Sousa \& Araújo, 2018).

As reações emocionais estressantes mais comuns são: choro, medo ou nervosismo, necessidade de lidar com incertezas, medo da morte, pensar em estar doente e não querer discutir sobre a doença. As crianças relatam estressores associados à interrupção nas rotinas e atividades diárias, como querer ser normal, sentir falta de casa, família, amigos, da escola e ficar restrito ao lar (Hildenbrand, Clawson, Alderfer \& Marsac, 2011). Para os adolescentes as múltiplas demandas de conviver com o câncer (incluindo suportar o diagnóstico e tratamentos, desconforto físico, limitações, dor, efeito na aparência e medos sobre o presente e o futuro) interferem nos aspectos psicológicos (Kurtz \& Abrams, 2011), em um período onde, geralmente busca-se a independência e a individualidade em relação aos pais e uma autoafirmação em relação aos pares.

Entre os principais quadros psicopatológicos apresentados evidencia-se a ansiedade, depressão e o transtornos de estresse pós-traumático (Akimana, Abbo, Balagadde-Kambugu \& Nakimuli-Mpungu, 2019; Kurtz \& Abrams, 2011; Steinmetz \& Tarquinio, 2018). Considerando a dimensão que o câncer infanto-juvenil assume na vida das crianças e dos familiares e cuidadores é comum 
que em algum momento do itinerário de tratamento algum destes sintomas possam aparecer na pessoa adoecida ou nas que estão convivendo com ela e a acompanhando no tratamento.

A ansiedade é um sintoma psicopatológico muitas vezes apresentado em decorrência das incertezas do processo terapêutico, às expectativas de reação ao tratamento realizado e ao futuro incerto frente ao adoecimento (Barbato, Antunes \& Lourenço, 2019). A depressão consiste em uma das psicopatologias que podem ser apresentadas quando a pessoa adoecida ou familiares e cuidadores não conseguem lidar com os eventos advindos do adoecimento e criar condições de enfrentamento diante de situações difíceis de serem vivenciadas (Akimana, Abbo, Balagadde-Kambugu \& Nakimuli-Mpungu, 2019; Kosir, Wiedemann, Wild \& Bowes, 2019).

A apresentação de sintomatologia depressiva durante o percurso do adoecimento por câncer na infância e adolescência pode surgir devido às condições impactantes e de difícil elaboração vivenciadas pelo adoecido e pelos familiares e cuidadores. Entre essas condições estão: não reação ao tratamento estipulado, impossibilidade de cura da doença, não aceitação do quadro de adoecimento, autoculpabilização pelo adoecimento, percepções equivocadas/distorcidas em relação a doença e ao tratamento, alterações nas relações familiares e sociais, isolamento, amputação de algum membro, mudanças corporais, alteração na percepção e autoestima, incapacidade de criar condições de enfrentamento, falta de suporte familiar para lidar com situações adversas, dificuldades de adaptação, dificuldade de retomada das atividades após finalização do tratamento, medo, incertezas, entre outros elementos; e contribuem para o aparecimento de sintomas depressivos.

O transtorno de estresse pós-traumático (TEPT) e seus sintomas associados é uma das psicopatologias mais evidenciadas em produções científicas sobre o adoecimento por câncer na infância e adolescência. O TEPT pode acometer tanto a pessoa adoecida, quanto seus familiares e cuidadores, mas não de forma sistemática. Na maior parte dos casos, envolve medo intenso, desamparo ou horror em resposta aos eventos traumáticos vivenciados endossados por sintomas de reviver (pensamentos intrusivos, sonhos, flashbacks) o corrido durante a doença, evitação (evitar lembretes do trauma, entorpecimento, afeto restrito) e excitação fisiológica (problemas de sono, hipervigilância, irritabilidade) (Steinmetz \& Tarquinio, 2018).

\section{Repercussões econômicas}

O câncer infantojuvenil incide diretamente nas questões econômicas da família, pois requer a disponibilização de recursos financeiros para arcar com custos das mais diversas naturezas. É importante ressaltar que o tratamento do câncer não é disponibilizado gratuitamente para todas as pessoas no mundo e, consequentemente, traz um impacto significativo para aqueles que precisam arcar com os gastos advindos da doença e como consequência há uma redução na renda das famílias que pode afetar o bem-estar dos sujeitos a ela pertencentes.

Um dos impactos diretos do câncer infantojuvenil está no emprego de um dos familiares ou cuidadores, como abandono ou perda do emprego, bem como mudanças nas jornadas de trabalho e mesmo afastamento prolongado (Roser, Erdmann, Michel, Winther \& Mader, 2019). Isso pode afetar financeiramente a família, demandando ajustes e suporte familiar.

$\mathrm{O}$ adoecimento por câncer pediátrico afeta a renda familiar a curto e longo prazo, uma vez que muitas famílias têm dificuldades para se ajustarem mesmo após a finalização do tratamento do adoecido, precisam mudar de emprego, cidade e até mesmo de residência (Santacroce \& Kneipp, 2019). No atendimento prestado nos serviços de saúde a equipe multiprofissional deve estar atenta para estes aspectos, acolher as demandas e realizar os encaminhamentos para outros órgãos governamentais, caso seja necessário. Em muitas ocasiões os familiares não manifestam abertamente as dificuldades financeiras enfrentadas, sendo necessário que a equipe de saúde fique atenta a estes aspectos, esteja aberta ao diálogo e indique que os profissionais do serviço social dos centros de tratamento podem auxiliar na resolução deste problema.

\section{Disparidades no acesso ao tratamento em contexto global}

O tratamento de câncer na infância e na adolescência irá requerer atenção em serviços de saúde especializados e com profissionais de diferentes especialidades. $\mathrm{O}$ acesso a cuidados de saúde adequados, de modo precoce e com qualidade é fundamental para otimizar a sobrevida, pois permite intervenções apropriadas enquanto a doença ainda está nos estágios iniciais (Dang-Tan \& Franco, 2007). As melhorias nos resultados do tratamento do câncer infantojuvenil podem diferenciar-se entre os países dependendo da estrutura e dos recursos oferecidos para 
as pessoas adoecidas, familiares e cuidadores. Quando o tratamento oportuno e ideal está disponível, acessível e completo, mais de $80 \%$ dos casos de câncer são curados (Arora, Challinor, Howard \& Israels, 2016), o que contrasta com a realidade de países pobres.

Apesar dos avanços, alguns desafios precisam ser considerados em relação aos índices de cura do câncer. O tratamento precisa de muitos especialistas, infraestrutura, equipamentos e medicamentos que elevam os custos e impossibilitam com que muitas crianças e adolescentes tenham acesso a centros especializados (Magrath, Steliarova-Foucher, Epelman, Ribeiro, Harif, Li, Kebudi, Macfarlane \& Howard, 2013). Os custos extremamente altos corroboram para a existência de uma desigualdade dramática na distribuição de recursos para o controle e tratamento em todo o mundo (Rodriguez-Galindo, Friedrich, Alcasabas, Antillon, Banavali, Castillo, Israels, Jeha, Harif, Sullivan, Quah, Patte, Pui, Barr \& Gross, 2015).

Neste cenário é possível perceber desigualdades em termos de acesso aos recursos de tratamento entre países com alta concentração de renda e países mais pobres. Tal situação provavelmente não terá alterações substanciais futuramente, pois estimativas afirmam que a lacuna na sobrevivência entre os países de alta renda e os países de baixa renda só aumentará nos próximos anos (Rodriguez-Galindo, Friedrich, Morrissey \& Frazier, 2013). Considerando que mais de $90 \%$ dos cânceres infantis ocorrem em países de baixa e média renda e as barreiras do sistema de saúde ao acesso e encaminhamento que resultam no subdiagnóstico do câncer infantil em muitos países (Ward, Yeh, Bhakta, Frazier \& Atun, 2019), nota-se o tamanho da problemática em questão.

Em países de baixa e média renda uma proporção significativa de crianças com câncer potencialmente curável não recebem o tratamento - nem mesmo cuidados paliativos - e a maioria morre (Magrath et al., 2013). De forma geral, as pessoas mais pobres têm menos acesso aos serviços de saúde.

A apresentação tardia do diagnóstico, o abandono do tratamento, a desnutrição, a falta de suporte e recursos necessários, infecções causadas por outras doenças graves, sistemas de prestação de cuidados de saúde ineficientes também acabam sendo fatores que interferem o sucesso do tratamento do câncer pediátrico (Rodriguez-Galindo, Friedrich, Morrissey \& Frazier, 2013). Ainda segundo esses autores, o grande desafio da oncologia pediátrica nas próximas décadas será traduzir os ganhos alcançados em ambientes de maior renda para as crianças em todo o mundo.

Em termos globais, a África é o continente com maior vulnerabilidade no sistema de atendimento devido a sistemas de saúde notoriamente deficientes e necessidades de saúde concorrentes que limitam o desenvolvimento de programas de controle do câncer (Rodriguez-Galindo et al., 2015). Ainda há poucos centros dedicados ao câncer infantil no continente, poucos oncologistas pediátricos e enfermeiras especializadas, crianças doentes são diagnosticadas tardiamente, o que consequentemente resulta em mais mortes no continente do que em outras partes do mundo (Stefan, Bray, Ferlay, Liu \& Parkin, 2017). Menos de $20 \%$ das crianças/adolescentes têm acesso ao tratamento curativo. Consequentemente, a sobrevivência de crianças com câncer na África é significativamente menor do que nos países desenvolvidos, onde a sobrevida global em 5 anos é de 70 a 80\% (Hadley, Rouma \& Saad-Eldin, 2012).

A Ásia constitui-se como uma região onde programas de atendimento estão sendo implementados com progressos significativos no desenvolvimento de iniciativas regionais no tratamento e controle do câncer (Rodriguez-Galindo et al., 2015). Contudo, devido à dimensão deste continente e a fatores sociais, políticos e econômicos, existem inúmeras discrepâncias entre os países que diferenciam o acesso da população ao tratamento.

A Europa, como concentra uma quantidade significativa de países de alta renda apresentou nas últimas décadas melhoras significativas nas possibilidades de acesso e nas formas de tratamento. Contudo, mesmo neste continente existem diferenças entre os países no acesso aos recursos de saúde e organização da assistência (Pritchard-Jones, Pieters, Reaman, Hjorth, Downie, Calaminus, Naafs-Wilstra \& Steliarova-Foucher, 2013).

O continente americano caracteriza-se por grandes disparidades em termos de acesso ao tratamento do câncer infantojuvenil, devido às dimensões territoriais e as diferenças econômicas existentes entre os países que o compõe. As diferenças nas condições de acesso a um tratamento adequado nesse continente acabam influenciando diretamente nos índices de sobrevida e cura dessa doença. Tais aspectos podem ser claramente evidenciados quando é observado que a mortalidade por câncer na América Latina caracteriza-se por ser duas vezes maior que na América do Norte (Magrath et al., 2013). Apesar destes dados, na América Latina 
nas últimas décadas têm sido realizados esforços para ampliar o atendimento à população por meio de centros especializados para o tratamento.

É importante ressaltar que mesmo no caso de países desenvolvidos e com bons sistemas de saúde para o atendimento de crianças e adolescentes com câncer, podem haver dicotomias em relação ao acesso ao tratamento. Tais aspectos podem ser observados ao considerar que nestes países existem desigualdades sociais, questões relacionadas a gênero, preconceito racial, preconceito, situações de xenofobia, não gratuidade no atendimento em centros especializados, entre outros aspectos que interferem no acesso aos recursos de saúde.

Outros fatores que podem explicar a diferença no sucesso do tratamento são: o diagnóstico da doença em estágios mais avançados, diagnóstico atrasados, diferentes biologias subjacentes, gravidade da toxicidade, abandono do tratamento, dificuldades para acessar e permanecer nos serviços formais de atendimento, inexistência de serviços de tratamento, custos financeiros do tratamento, entre outros. Fatores complexos específicos do local/cultura, onde a pessoa está inserida, acabam fazendo com que a atenção a situações de adoecimento por câncer não seja prioridade diante de outros problemas de saúde, atrasos na apresentação, estado nutricional ruim, custos de tratamento, disponibilidade inconsistente de medicamentos, falta de cuidados de suporte e abandono do tratamento (Arora, Eden \& Pizer, 2007).

Os aspectos anteriormente mencionados refletem as discrepâncias no acesso aos recursos de saúde no contexto global. A falta de acesso no tempo adequado, a medicamentos necessários ao tratamento e a centros de saúde especializados no atendimento ao câncer podem resultar em inúmeras complicações na vida das crianças e adolescentes adoecidos. É importante enfatizar que são poucos os países em que as pessoas possuem o acesso universal a um sistema público de saúde, demonstrando a necessidade da valorização do Sistema Único de Saúde brasileiro e dos profissionais que atuam nesse contexto.

\section{Repercussões do câncer na vida escolar}

$\mathrm{O}$ adoecimento por câncer na infância e adolescência incide diretamente nas condições educacionais, pois muitas vezes irá requerer o afastamento deste ambiente em decorrência dos tratamentos realizados e da constante hospitalização provocada pela doença. Além disso, deve-se ser considerado também que quando a criança apresenta uma condição crônica, o convívio com o hospital pode começar antes mesmo de ela ir à escola pela primeira vez e frequentemente, seu processo de escolarização não é iniciado, ou é interrompido temporariamente, ou abandonado (Silva, Xavier, Oliveira, Figueredo, Prado \& Aguiar Filho, 2016).

Embora algumas crianças consigam se manter na escola durante o tratamento do câncer, o que é importante para o seu desenvolvimento (Gomes, Lima, Rodrigues, Lima, \& Collet, 2013), o abandono das atividades escolares ainda é uma situação bastante comum. $\mathrm{O}$ afastamento das atividades escolares, embora em muitas situações seja necessário, não tem sido descrito como positivo para crianças e adolescentes por diferentes aspectos que vão desde a ruptura de vínculos, até os prejuízos em decorrência do afastamento dos estudos e conteúdos didáticos.

Em muitas situações a ausência na vida escolar representará o rompimento com todos os vínculos de amizade da criança e do adolescente, pode levar ao isolamento e consequentemente despertar sintomas de solidão diante da situação vivida. Muitas crianças e adolescentes relatam que não frequentam a escola em decorrência do câncer, de efeitos do tratamento, hospitalizações, consultas frequentes, entre outros (Leandro et al., 2018). Segundo os autores, tais aspectos levam-nas a terem sentimento de tristeza pela necessidade de abandono das atividades escolares e do convívio com os colegas de escola.

O retorno para a vida escolar geralmente caracteriza-se como sendo um desejo das crianças e adolescentes por câncer, pois representa a retomada da rotina e consequentemente o retorno para seus colegas e amigos. Voltar à escola representa voltar à vida normal e retomar os contatos sociais que tinha anteriormente, sendo neste retorno essencial ter a colaboração e compreensão dos colegas e professores (Enskär, Carlsson, Golsäter \& Hamrin, 1997).

Por outro lado, professores podem ficar receosos em terem que lidar com a situação de adoecimento por câncer da criança/adolescente devido a desinformação sobre a doença, efeitos do tratamento, o medo dos professores de não conseguirem atender o aluno dentro dos extremos de conservar limites e/ou exigências e atender suas necessidades especiais, a dificuldade de se posicionar frente aos outros alunos sobre o diagnóstico do câncer. Além disso, existe por parte da criança e da família o medo de enfrentarem mais uma situação nova geradora de exposição física e social (Silva \& Valle, 2005). 
Em algumas situações as crianças e adolescentes podem ficar vários meses afastadas do convívio com o ambiente de ensino, pois requerem atenção devido às condições de saúde que se encontram. Deste modo, sempre que possível é importante a busca por recursos alternativos para minimizar o impacto do afastamento da vida escolar. Também se mostra relevante que os centros de saúde ofereçam um espaço para atendimento educacional das crianças e adolescentes, uma vez que a vida escolar é um importante marco no desenvolvimento infantojuvenil e precisa ser considerado pelas equipes multidisciplinares que prestam atendimento para esse público.

\section{Considerações Finais}

O câncer na infância e adolescência caracteriza-se por ser um evento marcante e que acarreta rupturas no curso do desenvolvimento uma vez que trará implicações físicas, cognitivas e psicossociais que alterarão rotinas, atividades cotidianas, relações familiares, sociais e relacionais. O desenvolvimento humano na infância e adolescência é marcado por uma série de transformações em todos os aspectos da vida das pessoas que são profundamente transformados quando são perpassados por situações de adoecimento crônico, como é o caso do câncer infantojuvenil.

O câncer é um evento complexo e amplo, marcado por determinantes subjetivos e sociais e que tem se transformado ao longo dos anos em decorrência dos avanços nas formas de compreendê-lo e tratá-lo. Os avanços no campo da saúde têm possibilitado melhorias nos índices de sobrevivência, contudo, do ponto de vista psicológico este ainda é um evento traumático de ser vivenciado devido aos aspectos subjetivos socialmente construídos entorno da doença e as implicações que causam no organismo das pessoas, marcado por sofrimento, procedimentos invasivos e efeitos adversos que impactam nas condições e saúde global das pessoas e na qualidade de vida.

Evidenciar os aspectos psicossociais do câncer infanto-juvenil é um desafio pela complexa gama de eventos e situações que são apresentadas e atreladas a esta condição de adoecimento, marcada por implicações em todas as dimensões da vida das pessoas. As discussões apresentadas neste trabalho evidenciam parte dos elementos presentes no adoecimento por câncer e precisam ser considerados atentando-se para outros elementos que se fazem presentes na vida das pessoas e que estão atrelados ao contexto social e cultural de onde vivem e as particularidades de cada situação analisada.

Compreender as implicações psicossociais do câncer infantojuvenil é essencial para a prestação de um cuidado integral em saúde, uma vez que esse evento resultará em transformações no modo de vida da pessoa adoecida e afetará a dinâmica familiar. As crianças e adolescentes que chegam para atendimento nos serviços de saúde não chegam sozinhas. Elas trazem consigo histórias de vida particular, história de vida familiar, expectativas, medos, anseios, conflitos relacionais, raiva, esperança, fé, entre outros aspectos que muitas vezes interferirão no modo como irão reagir diante da doença, nas formas de enfrentamento que utilizarão, nos investimentos financeiros e emocionais em relação à doença e até mesmo no abandono do tratamento. Assim, é necessário principalmente aos profissionais de saúde conhecer a história de vida dos sujeitos atendidos e seus familiares/cuidadores e os aspectos psicossociais presentes no itinerário percorrido por estes desde o diagnóstico até o final do tratamento.

\section{Referências}

Akimana, B., Abbo, C., Balagadde-Kambugu, J. \& Nakimuli-Mpungu, E. (2019). Prevalence and factors associated with major depressive disorder in children and adolescents at the Uganda Cancer Institute. BMC Cancer, 19 (1), 466.

Arora, R. S., Eden, T. \& Pizer, B. (2007). The problem of treatment abandonment in children from developing countries with cancer. Pediatric Blood \& Cancer, 49 (7), 941-946.

Arora, R. S., Challinor, J. M., Howard, S. C. \& Israels, T. (2016). Improving care for children with cancer in low $\square$ and middle $\square$ income countries - a SIOP PODC initiative. Pediatric Blood \& Cancer, 63 (3), 387-391.

Barbato, K. B. D. A., Antunes, K. R. \& Lourenço, M. T. C. (2019). Reflexões sobre vivências da criança com câncer diante da morte. Revista da SBPH, 22 (1), 306-327.

Carrion-Plaza, A., Jaen, J. \& Montoya-Castilla, I. (2020). HabitApp: New play technologies in pediatric cancer to improve the psychosocial state of patients and caregivers. Frontiers in Psychology, 11, 157.

Dang-Tan, T. \& Franco, E. L. (2007). Diagnosis delays in childhood cancer. Cancer, 110 (4), 703-713.

Enskär, K., Carlsson, M., Golsäter, M. \& Hamrin, E. (1997). Symptom distress and life situation in adolescents with cancer. Cancer Nursing, 20 (1), 23-33.

Galligan, A. J. (2017). Childhood cancer survivorship and long-term outcomes. Advances in Pediatrics, 64 (1), 133-169.

Gomes, I. P., Lima, K. A., Rodrigues, L. V., Lima, R. A. G. \& Collet, N. (2013). Do diagnóstico à sobrevivência do câncer infantil: Perspectiva de crianças. Texto \& Contexto - Enfermagem, 22 (3), 671-679.

Hadley, L. G., Rouma, B. S. \& Saad-Eldin, Y. (2012). Challenge of pediatric oncology in Africa. Seminars in Pediatric Surgery, 21 (2), 136-141.

Hildenbrand, A. K., Clawson, K. J., Alderfer, M. A. \& Marsac, M. L. (2011). Coping with pediatric cancer: Strategies employed by children and their parents to manage cancer-related stressors during treatment. Journal of Pediatric Oncology Nursing, 28 (6), 344-354.

Kosir, U., Wiedemann, M., Wild, J. \& Bowes, L. (2019). Psychiatric disorders in adolescent cancer survivors: A systematic review of prevalence and predictors. Cancer Reports, 2 (3), e1168.

Kurtz, B. P., \& Abrams, A. N. (2010). Psychiatric aspects of pediatric cancer. Child and Adolescent Psychiatric Clinics, 19 (2), 401-421.

Leandro, T. A., Silva, V. M. D., Lopes, M. V. D. O., Guedes, N. G., Nunes, 
M. M., Sousa, T. M. D. \& Araújo, M. V. (2018). Conforto prejudicado em crianças e adolescentes com câncer. Revista Brasileira de Enfermagem, 71 (3), 934-941.

Magrath, I., Steliarova-Foucher, E., Epelman, S., Ribeiro, R. C., Harif, M., Li, C., Kebudi, R., Macfarlane, S. D. \& Howard, S. C. (2013). Paediatric cancer in low-income and middle-income countries. The Lancet Oncology, 14 (3), e104-e116.

Manoharan, N. \& O'Brien, T. (2020). Childhood cancer: Unique opportunities and inherent challenges. The Medical Journal of Australia, 212 (3), 110-111.

Pritchard-Jones, K., Pieters, R., Reaman, G. H., Hjorth, L., Downie, P., Calaminus, G., Naafs-Wilstra, M. C. \& Steliarova-Foucher, E. (2013). Sustaining innovation and improvement in the treatment of childhood cancer: lessons from high-income countries. The Lancet Oncology, 14 (3), e95-e103.

Rattan, S. (2020). A safe space to breathe. Advances in Mental Health, 18 (1), 50-61.

Rodriguez-Galindo, C., Friedrich, P., Morrissey, L. \& Frazier, L. (2013). Global challenges in pediatric oncology. Current Opinion in Pediatrics, 25 (1), 3-15.

Rodriguez-Galindo, C., Friedrich, P., Alcasabas, P., Antillon, F., Banavali, S., Castillo, L., Israels,T., Jeha, S., Harif, M., Sullivan, M. J., Quah, T. C., Patte, C., Pui, C., Barr, R. \& Gross, T. (2015). Toward the cure of all children with cancer through collaborative efforts: Pediatric oncology as a global challenge. Journal of Clinical Oncology, 33 (27), 3065.

Roser, K., Erdmann, F., Michel, G., Winther, J. F. \& Mader, L. (2019). The impact of childhood cancer on parents' socio $\square$ economic situation - A systematic review. Psycho $\square$ Oncology, 28 (6), 1207-1226.

Rother, E. T. (2007). Revisão sistemática X revisão narrativa. Acta Paulista de Enfermagem, 20 (2), v-vi.

Santacroce, S. J. \& Kneipp, S. M. (2019). Influence of pediatric cancer-related financial burden on parent distress and other stress $\square$ related symptoms. Pediatric Blood \& Cancer, 67 (3), e28093.
Siegel, R. L., Miller, K. D. \& Jemal, A. (2018). Cancer statistics, 2018. CA: A Cancer Journal for Clinicians, 68 (1), 7-30.

Silva, P. L. N., Xavier, G. C., Oliveira, V. V., Figueredo, M. L., Prado, P. F. \& Aguiar Filho, W. (2016). Câncer infantil: Vivências de crianças em tratamento oncológico. Enfermagem em Foco, 7 (3/4), 51-55.

Silva, G. M. \& Valle, E. R. M. (2005). A vivência da reinserção escolar de crianças com câncer: Estratégia de atuação do psicólogo. Revista Psicopedagogia, 22 (67), 2-13

Steinmetz, T. \& Tarquinio, C. (2018). Stress post-traumatique et liens d'attachement chez l'enfant atteint du cancer: Revue de littérature. L'Évolution Psycbiatrique, 83 (2), 235-250.

Stefan, C., Bray, F., Ferlay, J., Liu, B. \& Parkin, D. M. (2017). Cancer of childhood in sub-Saharan Africa. Ecancermedicalscience, 11, 755.

Steliarova-Foucher, E., Colombet, M., Ries, L. A., Moreno, F., Dolya, A., Bray, F., Hesseling,P., Shin,H. Y., Stiller, C. A. \& Tretarre, B. (2017). International incidence of childhood cancer, 2001-10: A population-based registry study. The Lancet Oncology, 18 (6), 719-731.

Ward, Z. J., Yeh, J. M., Bhakta, N., Frazier, A. L. \& Atun, R. (2019). Estimating the total incidence of global childhood cancer: A simulation-based analysis. The Lancet Oncology, 20 (4), 483-493.

Woodgate, R. L. (2006). Life is never the same: Childhood cancer narratives. European Journal of Cancer Care, 15 (1), 8-18.

Yi, J., Kim, M. A. \& Sang, J. (2016). Worries of childhood cancer survivors in young adulthood. European Journal of Oncology Nursing, 21, 113-119.

Submetido em: 26-1-2021

Aceito em: $18-10-2021$ 